\title{
Author Correction: Multi-region sequencing unveils novel actionable targets and spatial heterogeneity in esophageal squamous cell carcinoma
}

Ting Yan, Heyang Cui, Yong Zhou, Bin Yang, Pengzhou Kong, Yingchun Zhang, Yiqian Liu, Bin Wang, Yikun Cheng, Jiayi Li, Shixing Guo, Enwei Xu, Huijuan Liu, Caixia Cheng, Ling Zhang, Ling Chen, Xiaofei Zhuang, Yu Qian, Jian Yang, Yanchun Ma, Hongyi Li, Fang Wang, Jing Liu, Xuefeng Liu, Dan Su, Yan Wang, Ruifang Sun, Shiping Guo, Yaoping Li, Xiaolong Cheng, Zhihua Liu (D, Qimin Zhan \& Yongping Cui

Correction to: Nature Communications https://doi.org/10.1038/s41467-019-09255-1, published online 11 April 2019.

This Article contained errors in Fig. 1 and Supplementary Fig. 4. In Fig. 1. and Supplementary Fig. 4d the gels were inappropriately spliced together. For each experiment, multiple gels were prepared and the gels were probed in parallel. The figure legends have been updated to describe the parallel processing of the blots.

Additionally, in Supplementary Fig. 4d the colony formation image for ErbB4-E57K of the KYSE180 cells was inadvertently duplicated from the ErbB4-WT image. The image has now been replaced, while the experiment was repeated three times, images are only available from one experiment.

Finally, the original manuscript was published without the Source data for the histograms and gels. The Source data file now contains all the Source data supporting the study.

These errors have been corrected in the Pdf and HTML versions of the Article.

Published online: 12 November 2020

(i) Open Access This article is licensed under a Creative Commons Attribution 4.0 International License, which permits use, sharing, adaptation, distribution and reproduction in any medium or format, as long as you give appropriate credit to the original author(s) and the source, provide a link to the Creative Commons license, and indicate if changes were made. The images or other third party material in this article are included in the article's Creative Commons license, unless indicated otherwise in a credit line to the material. If material is not included in the article's Creative Commons license and your intended use is not permitted by statutory regulation or exceeds the permitted use, you will need to obtain permission directly from the copyright holder. To view a copy of this license, visit http://creativecommons.org/licenses/by/4.0/.

(C) The Author(s) 2020 